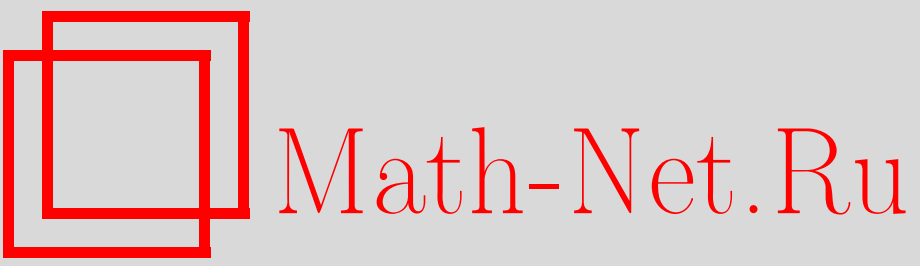

С. М. Натанзон, А. М. Пратусевич, Топология $m$-спинорных структур на римановых поверхностях, УМH, 2005, том 60, выпуск 2, 169-170

DOI: https://doi.org/10.4213/rm1419

Использование Общероссийского математического портала Math-Net.Ru подразумевает, что вы прочитали и согласны с пользовательским соглашением

http://www . mathnet.ru/rus/agreement

Параметры загрузки:

IP : 3.85 .183 .62

26 апреля 2023 г., 09:24:09 


\title{
ТОПОЛОГИЯ $m$-СПИНОРНЫХ СТРУКТУР НА РИМАНОВЫХ ПОВЕРХНОСТЯХ
}

\author{
C. М. НАТАНЗОН, А. М. ПРАТУСЕВИч
}

1. Классические спинорные расслоения на компактных римановых поверхностях (тета-характеристики) активно используются в геометрии со времен Римана [1]. Их современная классификация и интерпретация как линейных расслоений, квадрат которых изоморфен кокасательному расслоению, предложены Атьей [2] и Мамфордом [3]. Они связали множество (классических) спинорных расслоений на римановой поверхности $P$ с аффинным пространством функций Арфа (в смысле [4]), т.е. квадратичных форм $H_{1}(P, \mathbb{Z} / 2 \mathbb{Z}) \rightarrow \mathbb{Z} / 2 \mathbb{Z}$ (относительно индекса пересечения на $\left.H_{1}(P, \mathbb{Z} / 2 \mathbb{Z})\right)$. Топологические аспекты исследованы в [5]. Классификация классических спинорных структур на некомпактных поверхностях дана в [6]. В настоящей статье мы анонсируем соответствующие резултаты для $m$-спинорных расслоений. Теория для тора и цилиндра проста и описана в [7]. Поэтому далее мы рассматриваем лишь гиперболические римановы поверхности.

2. На компактных римановых поверхностях $m$-спинорное расслоение - это линейное расслоение, $m$-я степень которого изоморфна кокасательному расслоению. На некомпактной поверхности $P$ спинорная структура $E \rightarrow P$ определяется с помощью униформизации $P=\mathbb{H} / \Gamma$, где $\mathbb{H}-$ верхняя полуплоскость комплексной плоскости, а $\Gamma$ - фуксова группа, состоящая из ее гиперболических и параболических автоморфизмов. В этом случае $m$-спинорная структура определяется как фактор-расслоение тривиального расслоения $\mathbb{H} \times \mathbb{C} \rightarrow \mathbb{H}$ под действием группы $\Gamma$, где $g \cdot(z, t)=\left(g(z), \delta_{g}(z) \cdot t\right), \delta_{g_{2} g_{1}}=\left(\delta_{g_{2}} \circ g_{1}\right) \cdot \delta_{g_{1}}$ и $\delta_{g}^{m}=\left(g^{\prime}\right)^{-1} \cdot m$-спинорные структуры играют важную роль в математической физике [8] и теории особенностей [9].

Мы связьваем с $m$-спинорной структурой на $P$ функцию на множестве гомотопических классов простых контуров на $P$.

ОПРЕДЕЛЕнИЕ. Пусть $\pi_{1}^{0}(P, p) \subset \pi_{1}(P, p)$ - подмножество фундаментальной группы, состоящее из элементов, представимых простьми контурами. Отображение $\sigma: \pi_{1}^{0}(P, p) \rightarrow \mathbb{Z} / m \mathbb{Z}$ назовем $m$-функиией Арфа, если

1) $\sigma\left(b a b^{-1}\right)=\sigma(a)$

2) $\sigma\left(a^{-1}\right)=-\sigma(a)$

3) $\sigma(a b)=\sigma(a)+\sigma(b)$, если $a$ и $b$ можно представить простыми контурами таким образом, чтобы точка $p$ была их единственной общей точкой и их индекс пересечения не 0 ;

4) $\sigma(a b)=\sigma(a)+\sigma(b)+1$, если: а) $a$ и $b$ можно представить простыми контурами таким образом, чтобы точка $p$ была их единственной общей точкой и их индекс пересечения равен 0 ; б) ориентированные контуры $a, b$ и $(a b)^{-1}$ свободно гомотопны попарно не пересекающимся простьм контурам $\tilde{a}, \tilde{b}, \tilde{c}$, ориентации которых индуцированы комплексной структурой сфферы с 3 дырами, которую они отсекают от $P$.

Теорема 1. Пусть $P$ - риманова поверхность рода $g$ с $n$ проколами и $k$ дырами. Тогда:

1) существует естественное взаимно однозначное соответствие меэду т-спинорными структурами на $P$ и т-функииями Арфа на $P$;

2) множество $\Sigma$ всех $m$-функиий Арфа на $P$ состоит из $m^{2 g+n+k-\chi}$ әлементов, где $\chi=1$, если $n+k>0$, и $\chi=0$ в противном случае;

3) пара $\sigma_{1}, \sigma_{2} \in \Sigma$ порождает линейный функционал $\sigma_{1}-\sigma_{2} \in H^{1}(P, \mathbb{Z} / m \mathbb{Z})$. Более mого, $\Sigma=\sigma+H^{1}(P, \mathbb{Z} / m \mathbb{Z})$, где $\sigma \in \Sigma$.

3. Опишем теперь простейшие топологические инварианты $m$-функций Арффа $\sigma$. K ним мы отнесем род $g$ поверхности $P$ и число $s_{i}$ (соответственно $t_{i}$ ) проколов (соответственно дыр) поверх-

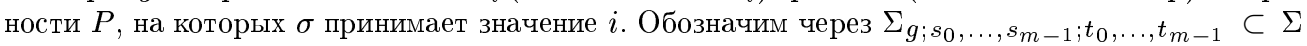

Первьй автор поддержан грантами РФФИ-04-01-00762, НШ-1972.2003.1; второй автор поддержан SFB 611 (DFG). 
соответствующее множество $m$-функций Арфа. Из определения $m$-функций Арфа сразу следует, что группа Mod гомотопических классов сохраняющих ориентацию автогомеоморфизмов поверхности $P$ естественно действует на множестве $\Sigma_{g ; s_{0}}, \ldots, s_{m-1} ; t_{0}, \ldots, t_{m-1} \subset \Sigma$.

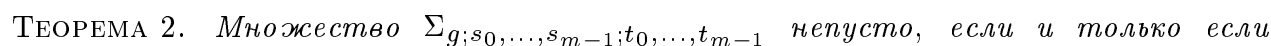
$\sum_{0 \leqslant i \leqslant m-1} i\left(s_{i}+t_{i}\right)=(2-2 g)-(n+k)(\bmod m)$. Действие группы $\operatorname{Mod}$ на непустом множестве $\Sigma_{g ; s_{0}}, \ldots, s_{m-1} ; t_{0}, \ldots, t_{m-1}$ транзитивно, если и только если выполнено хотя бь одно из следующих условий: 1) $m$ нечетно; 2) существует четное число $i$ такое, что $s_{i}+t_{i} \neq 0$. В противном случае группа Mod имеет на $\Sigma_{g ; s_{0}, \ldots, s_{m-1} ; t_{0}, \ldots, t_{m-1}}$ ровно 2 орбитьи.

4. Используем теперь $m$-функции Арфа для изучения пространства $M_{g, n, k}^{m}(2 g+n+k>2)$ модулей $m$-спинорных структур. По определению - это пространство пар $(P, \mu)$, где $\mu-m$-спинорная структура на римановой поверхности $P$ рода $g$ с $n$ проколами и $k$ дырами. Для $m=2$ его топология исследована в [6], [10]. Для произвольного $m$ пространство $M_{g, n, k}^{m}$ естественно распадается на подмножества $M_{g ; s_{0}}^{m}, \ldots, s_{m-1} ; t_{0}, \ldots, t_{m-1}$, отвечающие простейшим топологическим инвариантам соответствующих $m$-функций Арфа. Из теоремы 2 сразу следует

Теорема 3. Множество $M_{g ; s_{0}, \ldots, s_{m-1} ; t_{0}, \ldots, t_{m-1}}^{m}$ непусто, если и только если $\sum_{i=0}^{m-1} i\left(s_{i}+t_{i}\right)=(2-2 g)-(n+k)(\bmod m)$. Оно связно, если и только если выполнено хотя бы одно из следующих условий: 1) $m$ нечетно; 2) существует четное число $i$ такое, что $s_{i}+t_{i} \neq 0$. В противном случае множество $M_{g ; s_{0}, \ldots, s_{m-1} ; t_{0}, \ldots, t_{m-1}}^{m}$ распадается на 2 компоненты связности.

Для $k=0$ это утверждение другим (алгебро-геометрическим) методом доказано в [7].

Используя теоремы 1, 2 и теорему Фрике-Клейна [11] в форме [10], можно доказать следующее утверждение.

ТЕОРемА 4. Компонента связности пространства $M_{g, n, k}^{m}(2 g+n+k>2)$ гомеоморфна $\mathbb{R}^{d} / G$, где $d=6 g+2 n+3 k-6 u G \subset \operatorname{Mod}-$ дискретная группа.

\section{СПИСОК ЛИТЕРАТУРЫ}

[1] B. Riemann. Gesammelte mathematische Werke und wissenschaftlicher Nachlaß. New York: Dover, 1953. [2] M. F. Atiyah // Ann. Sci. École Norm. Sup. (4). 1971. V. 4. P. 47-62. [3] D. Mumford // Ann. Sci. École Norm. Sup. (4). 1971. V. 4. P. 181-192. [4] Б. А. Дубровин, С. П. Новиков, А. Т. Фоменко. Современная геометрия: Методы и приложения. M.: Наука, 1986. [5] D. Johnson // J. London Math. Soc. (2). 1980. V. 22. № 2. P. 365-373. [6] С. М. Натанзон // Матем. заметки. 1989. Т. 45. №4. С. 111-116. [7] Т. J. Jarvis // Internat. J. Math. 2000. V. 11. № 5. P. 637-663. [8] E. Witten // Topological Methods in Modern Mathematics (Stony Brook, 1991). Houston: Publish or Perish, 1993. P. 235-269. [9] I. V. Dolgachev // Math. Ann. 1983. V. 265. P. 529-540. [10] C. М. Натанзон. Модули римановых поверхностей, вещественных алгебраических кривых и их супераналоги. М.: МЦНМО, 2003. [11] R. Fricke, F. Klein. Vorlesungen über die Theorie der automorphen Funktionen. B. I: Die gruppentheoretischen Grundlagen. B. II: Die funktionentheoretischen Ausführungen und die Andwendungen. New York: Johnson Reprint Corp., 1965.

Московский государственньй университет, Московский независимьй университет,

Институт теоретической и эксперименталњной физики; Mathematisches Institut, Universität Bonn

E-mail: natanzon@mccme.ru; anna@math.uni-bonn.de
Представлено С. П. Новиковым Принято редколлегией 25.01.2005 